\title{
Efficacy of Heparinoid Cream Containing Pseudo-Ceramide for Remission of Atopic Dermatitis
}

\author{
Megumi Matsuoka' \\ Keita Okoshi' \\ Shotaro Ito (D) \\ Takuji Kume ${ }^{2}$ \\ Tsuyoshi Seki' \\ Takahiro Nishizaka' \\ Joji Okada ${ }^{3}$ \\ Azumi Nagasawa' \\ Makoto lijima' \\ Masatoshi $\mathrm{Abe}^{4}$ \\ Osamu Nemoto 4
}

'Health \& Wellness Products Research, Kao Corporation, Sumida-ku, Tokyo, Japan;

${ }^{2}$ Analytical Science Research, Kao Corporation, Wakayama, Japan; ${ }^{3}$ Skin Care Products Research, Kao Corporation, Sumida-ku, Tokyo, Japan; ${ }^{4}$ Sapporo Skin Clinic, Sapporo, Hokkaido, Japan
Correspondence: Azumi Nagasawa Health \& Wellness Products Research, Kao Corporation, 2-I-3, Bunka, Sumida-ku, Tokyo, I3I-850|, Japan

Tel +8I-3-5630-9443

Fax +8I-3-5630-9342

Email nagasawa.azumi@kao.com
Purpose: Atopic dermatitis (AD) is characterized by chronic inflammation, which frequently recurs, is exacerbated, and enters remission. A maintenance remission period is important for AD patients. We developed a formulation for use during $\mathrm{AD}$ remission, containing heparinoid and pseudo-ceramide that forms a lamellar structure. We evaluated the allergen permeability and examined the formulation's efficacy in maintaining remission in patients with AD.

Materials and Methods: Seventeen AD patients applied a cream containing $0.3 \%$ heparinoid and pseudo-ceramide (test cream group, $\mathrm{n}=10$ ), or a general cream containing $0.3 \%$ heparinoid (control cream group, $\mathrm{n}=7$ ) to their arm for four weeks after inducing remission with the application of a steroid cream for two weeks.

Results: The lamellar structure of the test cream was confirmed with small- and wide-angle $\mathrm{x}$-ray scattering analysis and observation by transmission electron microscopy. The test cream inhibited the penetration of V8 protease significantly compared to the control cream in vitro. According to $\mathrm{AD}$ severity score by dermatologists, the effects remission maintenance of the test cream group were comparable to those of the control cream group. However, the test cream group had a significantly increased skin hydration value compared to the control cream group. A significant decrease in transepidermal water loss, an indicator of skin barrier function, was shown in the test cream group compared to the control cream group.

Conclusion: The cream with lamellar structures containing heparinoid and pseudoceramides may inhibit allergen penetration. Moreover, skin properties improved during the remission period; thus, the formulation we developed was suitable for use during the AD remission period.

Keywords: atopic dermatitis, barrier, lamellar, heparinoid, ceramide, skin hydration, transepidermal water loss

\section{Introduction}

Atopic dermatitis (AD) is a disease caused mainly by eczema with pruritus, which repeatedly recurs accompanied by exacerbation and a remission period. ${ }^{1}$ The skin of AD patients has a decreased barrier function, leading to antigen sensitization and inflammation. ${ }^{2}$ This is caused by the facile invasion of stimulating substances. ${ }^{3}$ Moreover, a decrease in skin hydration of the stratum corneum leads to increased susceptibility to itchiness following stimulation. Scratching leads to further deterioration of the barrier function. ${ }^{4}$

During the remission period, AD patients are prone to inflammation relapse even from mild stimulation. AD skin has low barrier function of the stratum 
corneum in both the lesional area and the non-lesional area, ${ }^{5}$ and improving the skin barrier function is essential for preventing the relapse of inflammation. Decreased barrier function makes it easier for external stimulatory compounds, such as hapten and protein allergens, to invade. Further, the release of aggravating factors, such as V8 protease from the bacteria Staphylococcus aureus, occurs in AD patients. ${ }^{6}$ The invasion of an external allergen triggers inflammation. ${ }^{7}$ The invasion of an allergen produces $\mathrm{TH}$ 2-type (Th2) cytokines, like interleukin-4 (IL-4), interleukin-13 (IL-13), interleukin-31 (IL-31), and thymic stromal lymphopoietin (TSLP), ${ }^{8-10}$ thereby inducing an itching sensation. Scratching the itchy skin leads to a further decline in barrier function, creating a loop, called itch-scratch cycle. ${ }^{4}$

The treatment of AD is based on the three basic principles: drug treatments to alleviate inflammation, skincare, and removal of deteriorating factors. ${ }^{1}$ The drug treatment is expected to reduce inflammation and itching with the use of anti-inflammatory agents and anti-histamine, respectively. On the other hand, in the remission period without inflammation, the importance of skincare increases. Although mucopolysaccharides, like heparinoids, have been widely used in skincare to improve dry skin, ${ }^{11,12}$ they have little effect on the immediate improvement of the barrier function. ${ }^{13}$ Therefore, it is necessary to quickly strengthen the barrier function of the skin, to prevent the relapse of AD symptoms and prolong the remission period.

Ceramide, one of the main components of intercellular lipids, is important for the barrier function of the stratum corneum. $^{14-16}$ It has been found that ceramide levels are lower in $\mathrm{AD}$ patients than in healthy people. ${ }^{17-19}$ Intercellular lipids express barrier function by forming lamellar structures. ${ }^{20-22}$ We hypothesize that the inclusion of a lamellar structure similar to intercellular lipids on the skin may aid in improving the barrier function of $\mathrm{AD}$ and suppressing itchiness.

Therefore, we developed a moisturizer containing pseudo-ceramide and a heparinoid that forms a lamellar structure, and verified the effect on the remission period of AD.

\section{Materials and Methods}

\section{Test Cream}

The test cream was an $\mathrm{O} / \mathrm{W}$ (oil-in-water)-type cream formulation with $0.3 \%$ heparinoid and $2.5 \%$ synthetic pseudo-ceramide. The cream contains P-Cer [ $N$-(3-hexadecyloxy-2-hydroxypropyl)- $N$-2-(hydroxyethyl) hexadecanamide], stearyl alcohol, stearyl monoglyceride, glycerin, and water. Pseudo-ceramide, stearyl alcohol, and stearyl monoglyceride were made into appropriate compositions to formulate a cream with a lamellar structure. The control cream was a commercial $\mathrm{O} / \mathrm{W}$-type emulsion cream containing $0.3 \%$ heparinoid with stearyl alcohol, glycerol monostearate, polyoxyl stearate, glycerin, and water. An $\mathrm{O} / \mathrm{W}$-type commercial steroid cream containing $0.15 \%$ prednisolone valerate acetate, based on petroleum jelly, was used during the pre-treatment period.

\section{Observation by Transmission Electron Microscopy (TEM)}

The structures of the dry coating of the heparinoid barrier cream and control cream were examined according to the following procedures. Each cream was applied onto a polyethylene terephthalate (PET) film using a coater (120 $\mu \mathrm{m}$ thickness) and dried for $3 \mathrm{~h}$. Thereafter, they were cut into $5 \mathrm{~mm}$ squares, stained and fixed for $24 \mathrm{~h}$ at $23^{\circ} \mathrm{C}$ with osmium tetroxide. For ultramicrotome, we made ultra-thin sections with a thickness of $50-100 \mathrm{~nm}$ at $-80^{\circ} \mathrm{C}$ using Cryospec UC-6 (Leica Microsystems, Wetzlar, Germany). The sections were collected with a $2.3 \mathrm{M}$ sucrose ball and placed on a grid (non-carbon-reinforced film-coated 200 mesh) and were thereafter washed and dried. We used H-7650 (Hitachi, Tokyo, Japan) transmission electron microscope, and measurements were taken with an acceleration voltage between 80 and $100 \mathrm{kV}$. Furthermore, CCD (1024 x 1024 pixel) was used for detection.

\section{Small-Angle/Wide-Angle X-Ray Scattering Measurements}

The small- and wide-angle X-ray scattering apparatus (SAXSess $\mathrm{mc}^{2}$, Anton Paar, Graz, Austria) was used to evaluate the dry coating produced by the test and control creams. To sample the dry coating, the test and control creams were applied to a PET resin overhead projector film using a coater (120 $\mu \mathrm{m}$ thickness), dried for $3 \mathrm{~h}$ at $22^{\circ} \mathrm{C}$ and $40 \%$ relative humidity $(\mathrm{RH})$, and scratched off with a spatula before being transferred into the sample cell. For the sample cell, we used a paste cell dedicated for use with SAXSess $\mathrm{mc}^{2}$. An imaging plate dedicated for use with SAXSess $\mathrm{mc}^{2}$ was used as the detector for X-ray diffraction patterns. In terms of the conditions for measurement, the wavelength of the $\mathrm{X}$-rays were $\lambda=$ $0.1542 \mathrm{~nm}$, sample-detector distance was set to $259.2 \mathrm{~mm}$, and the measurement temperature was set to $25^{\circ} \mathrm{C}$. The exposure time was set to between 5 and 60 minutes, depending on the 
balance between the irradiation limit of the imaging plate and detection sensitivity (SN ratio). The two-dimensional diffraction image obtained by exposing the imaging plate to light was used to create a one-dimensional diffraction profile using the SAXSess $\mathrm{mc}^{2}$-attached software (SAXSquant 2D, SAXSquant), (Anton Paar, Graz, Austria). Period $d$ [nm] of the lamellar structure observed on X-ray scattering was determined from the peak position $q=2 \pi / \mathrm{d}\left[\mathrm{nm}^{-1}\right]$, where $q$ is the scattering vector.

\section{In vitro Allergen Penetration Experiment}

The allergen penetration experiment was conducted according to the following procedures: $10 \mathrm{mg} / \mathrm{cm}^{2}$ of the heparinoid barrier cream or control cream was applied on a $12 \%$-NON-SDS-PAGE (sodium dodecyl sulfate-polyacrylamide gel electrophoresis) gel (Tefco, Tokyo, Japan), and dried for $18 \mathrm{~h}$ at room temperature. Next, a filter paper (diameter $6 \mathrm{~mm}$ ) soaked in $20 \mu \mathrm{L}$ of $2 \mathrm{mg} / \mathrm{mL} \mathrm{V} 8$ protease (27 kD) aqueous solution (Source: Staphylococcus aureus) (FUJIFILM, Tokyo, Japan) was placed on top and allowed to stand for $6 \mathrm{~h}$ at $32^{\circ} \mathrm{C}$ and $95 \% \mathrm{RH}$. The filter paper was then removed, and the dried cream was removed by washing with water. The V8 protease which permeated into the acrylamide gel through the dried cream was stained using Coomassie Brilliant Blue (CBB) solution (Integrale, Tokushima, Japan). The water-washed gel was immersed into the $\mathrm{CBB}$ solution for 60 minutes with shaking and thereafter washed with water for 30 minutes, this was repeated twice. The quantitation of V8 protease stained with CBB was performed using the GS-900TM Calibrated Densitometer (Bio-Rad, California, United States).

\section{Efficacy Test}

The protocol of this randomized, double-blind human study was approved by the Institutional Review Board of the Kao Corporation (Tokyo, Japan) and Sapporo Skin Clinic
(Sapporo, Japan) (UMIN registration no. 000025722). This study was performed in accordance with the Declaration of Helsinki from December 2016 to March 2017 in Sapporo, Japan. Written informed consent was obtained from all participants prior to the start of the study.

\section{Subjects and Evaluation Flow}

To verify the effect of the cream with a lamellar structure on the remission period of $\mathrm{AD}$, Japanese females with mild to moderate symptoms of $\mathrm{AD}$ on their inside of arms were prescreened via visual observation and medical interviews conducted by dermatologists. Of the 33 subjects that were prescreened, 18 were deemed eligible by the investigator and study director.

After screening, 18 subjects were assigned to two groups. Eleven subjects aged 23-47 years old (mean, $33.7 \pm 9.1$ ) were assigned to the $0.3 \%$ heparinoid test cream group (test cream group), the remaining seven subjects aged 21-47 years old (means, $32.9 \pm 9.7$ ) were assigned to the $0.3 \%$ heparinoid control cream group (control cream group).

After a 1-week $(-3 \mathrm{~W})$ washout period, a steroid cream was applied for two weeks as a pre-treatment period. Then, application of the test formulation was initiated. The test site was visually evaluated, measured using various equipment, and photographed every week, from week 0 to week 4 (Figure 1). If skin condition markedly worsened during this period, the subject received appropriate treatment from a dermatologist.

\section{Application of Test or Control Cream}

Approximately $0.5 \mathrm{~g}$ of test or control cream was applied to the arm twice daily, once in the morning and once at night, for 4 weeks. During the study, if abnormalities were observed at the application site, a physician provided appropriate treatment and drug use was discontinued as

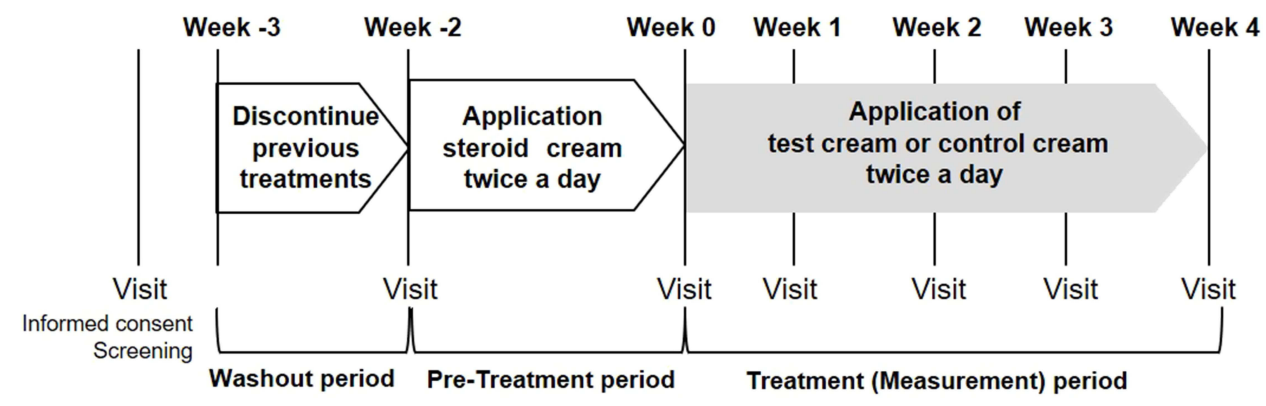

Figure I Test design.

Notes: After a I-week washout period, steroid cream was applied for two weeks as a pre-treatment. The test or control creams were applied twice a day for 4 weeks. 
necessary. The application of other moisturizing agents or topical agents to the test site during the study period was prohibited.

\section{Safety and Efficacy Assessments}

$\mathrm{AD}$ severity score on the arm was rated from 0 to 4 , including intermediate grades, for a total of nine grades as follows: 0 (absent), 0.5 (slight), 1 (mild), 1.5 (between mild and moderate), 2 (moderate), 2.5 (between moderate and severe), 3 (severe), 3.5 (between severe and very severe), 4 (very severe). The severity score was based on the severity classification (simple method) of AD developed by the advisory committee of $\mathrm{AD}$ severity classification of Japanese Dermatological Association. ${ }^{23}$ Assessment of efficacy was performed by a dermatologist. Subjects were asked to write in a journal every day during the study period regarding the presence or absence of itching anytime during in the day or night.

Adverse events were recorded throughout the study period. The severity of the adverse events was judged by dermatologist.

\section{Skin Measurements}

All instrumental measurements were conducted in an airconditioned room at $20 \pm 2{ }^{\circ} \mathrm{C}$, and $45 \pm 5 \% \mathrm{RH}$ after acclimatization for $20 \mathrm{~min}$. The same place (inside of the forearms) was measured every time by making a transparent sheet showing the measurement site for each subject. The sheet was applied on their arms, and the measurement site was marked before the acclimatization. Transepidermal water loss (TEWL) on the arm was measured using a Tewameter TM300 (Courage + Khazaka Electronic, Koln, Germany). The moisture content of the stratum corneum was measured with a SKICON-200EX (IBS, Hamamatsu, Japan).

\section{Statistical Analyses}

In the allergen penetration experiments, multiple comparison tests were performed according to the Tukey-Kramer method. In the application study for AD subjects, we determined the analysis population of subjects based on compliance status, which was defined by usage of test products (\%) for more than $80 \%$ of the time, and conducted significance testing. For significance testing, the results of AD severity score were analyzed using the Steel-Dwass multivariate test between the two groups. We subtracted the skin property measurements recorded on the start date (Week 0) from the skin property measurements recorded at the end of the study to determine the changes $(\Delta)$. The significance testing for changes to the skin properties was carried out according to Steel-Dwass multivariate testing.

The significance level was set at $95 \%$ confidence limits $(\mathrm{p}<0.05)$. Statistical analysis was performed using IBM SPSS Statistics version 19 (IBM, New York, United States) and Ekuseru-Toukei 2012 (Social Survey Research Information, Tokyo, Japan).

\section{Results}

\section{Structural Analysis of Test and Control Cream}

We used TEM observation to confirm whether the pseudoceramide-containing test cream forms the desired lamellar structure. A layered structure was observed in the test cream (Figure 2), however, in the control cream a layered structure was not observed (Data was not shown).

As TEM observation showed lamellar structure in the test cream, we used small- and wide-angle X-ray scattering to confirm the detail of the lamellar structure. In the test cream, after application and drying, a peak was observed at $q=0.63 \mathrm{~nm}^{-1}$ Figure $3 \mathrm{~A}$ (i). The results showed that the test cream had a lamellar structure of approximately $10 \mathrm{~nm}$ containing an aqueous phase. A peak was also observed at $q=15.4 \mathrm{~nm}^{-1}$ Figure 3A (ii). The results indicated that the dry coating film of the test cream had an $\alpha$-gel structure. Conversely, in the control cream a peak equivalent to the period of the layered structure was not detected Figure 3B (iii). Furthermore,

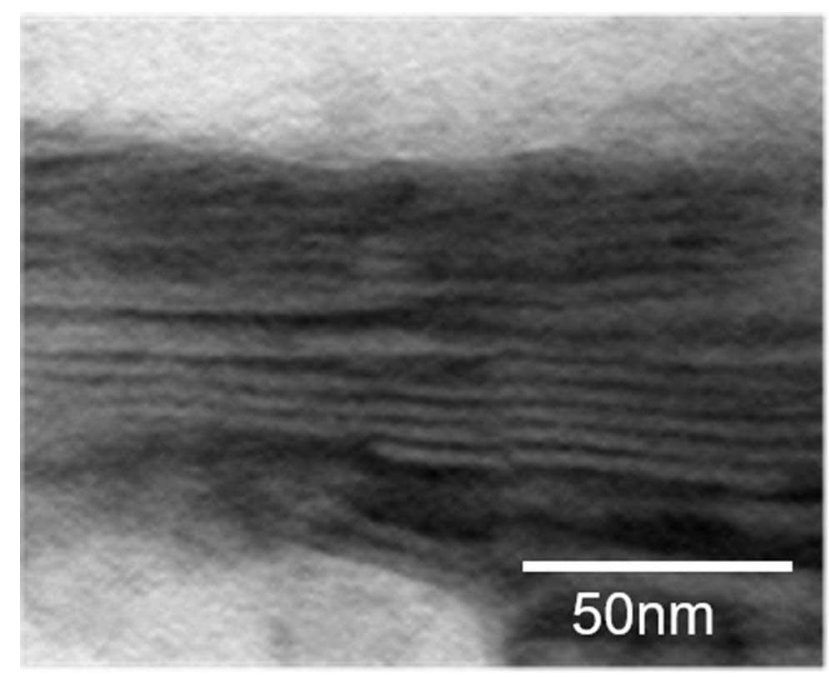

Figure 2 Lamellar structure image of test cream by transmission electron microscopy.

Notes: The layered structure was observed after coated on the film. 


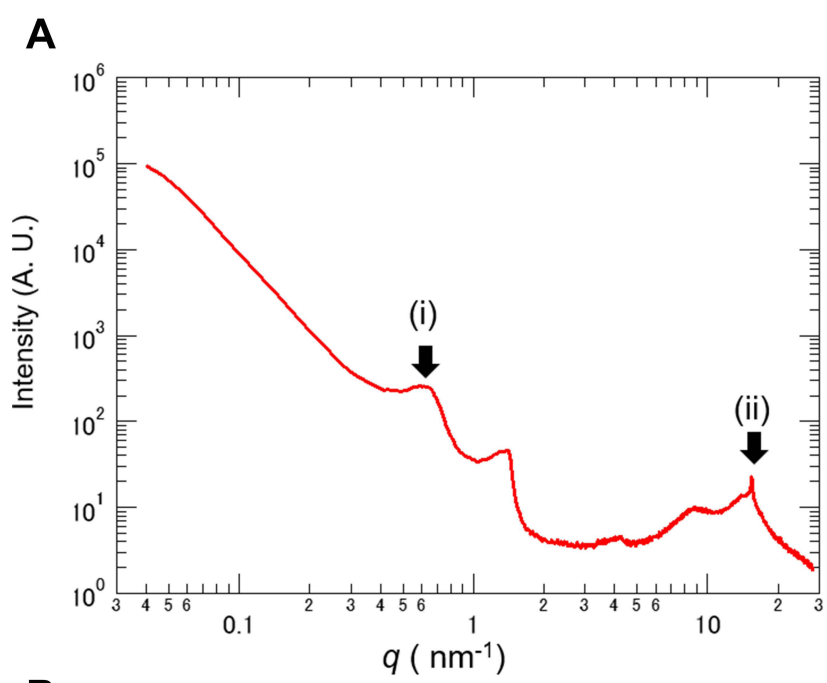

B

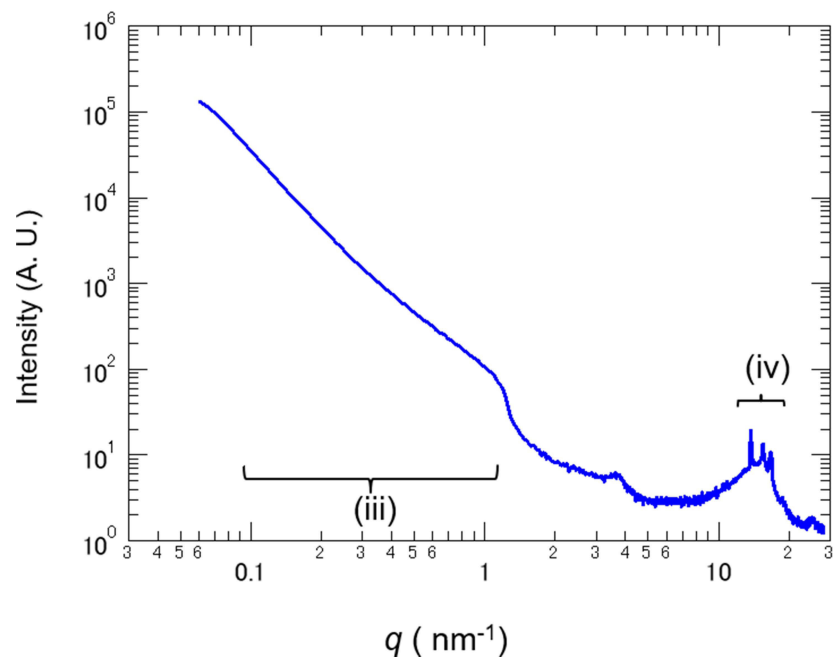

Figure 3 Small- and wide-angle $X$-ray scattering analysis of test and control cream. Notes: (A) The test cream showed a peak at scattering vector $q=0.63 \mathrm{~nm}^{-1}$ (i) and $q=15.4 \mathrm{~nm}^{-1}$ (ii). The lamellar period of the test cream was calculated as 10 $\mathrm{nm}$. (B) The control cream did not exhibit a clear peak within the $q=0.1-1 \mathrm{~nm}^{-1}$ range (iii). Multiple peaks derived from crystal structures were observed in the control cream (iv).

multiple peaks derived from the crystal structure were observed in the control cream Figure 3B (iv). These results confirmed that there was a lamellar structure in the test cream after application.

\section{Allergen Penetration Experiment}

The ability to suppress allergen penetration in a dry coating film was evaluated. V8 protease from Staphylococcus aureus was used as an allergen to evaluate the level of penetration. The level of penetration was calculated as a percentage. The penetration was $100 \%$ for no application (uncoated). The penetration of the control cream was $48.6 \% \pm 15.0$ (Figure 4). The penetration of the test

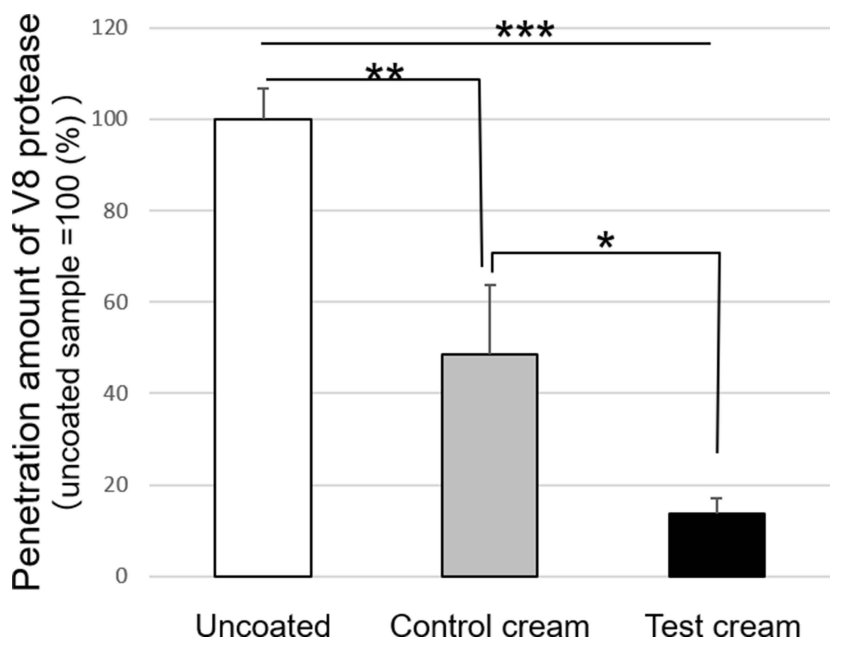

Figure 4 Penetration of V8 protease via test and control cream after application. Notes: The V8 protease which permeated into the acrylamide gel through the cream was stained using CBB solution. The level of penetration of the test cream were significantly lower than those of uncoated and control cream (mean $\pm \mathrm{SD}$, *p $<0.05$, ** $<<0.01$, *** $\mathrm{p}<0.00$ I, Tukey-Kramer method).

Abbreviations: $\mathrm{CBB}$, Coomassie brilliant blue; SD, standard deviation.

cream was $13.8 \% \pm 3.4$, which was significantly lower than that of uncoated or the control cream application. This indicates that the test cream with the lamella structure significantly suppressed the penetration of proteins, such as allergens.

\section{Efficacy Test}

One subject in the test cream group was excluded from due to lack of compliance with the study protocol. Another subject in the test cream group could not attend the week 3 visit because of fever. Therefore, we finally evaluated the efficacy and skin properties of nine to ten subjects for the test cream group and seven subjects for the control cream group. In the test cream group, the number of subjects for the evaluation of severity assessment and skin property measurements was ten subjects at weeks $0,1,2$, and 4 and nine at week 3.

\section{Efficacy Assessment}

The severity scores for $\mathrm{AD}$ were assigned in nine stages, from 0 : absent to 4 : very severe. The severity score after the washout period was $1.32 \pm 0.80$ (minimum of 0 , maximum of 3). At the start of the study, when the skin condition was temporarily improved using steroids (Week 0 ), the severity score for the heparinoid barrier cream group was $0.1 \pm 0.32$ and that of the control cream group was $0.07 \pm 0.19$ (Figure 5). Two weeks after the start of the study, in both groups, more than $50 \%$ of subjects had a score of 0.5 : slight 


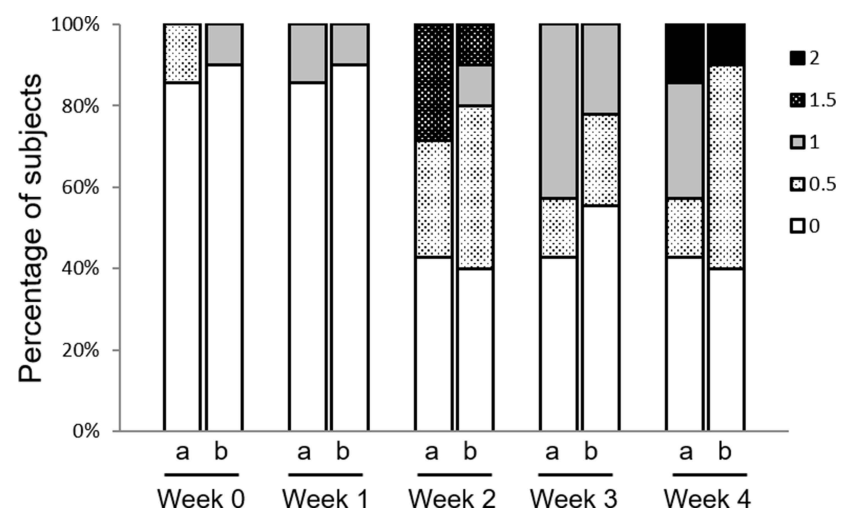

Figure 5 Changes in severity assessment after cream application. Notes: AD severity score was rated from 0 to 4 , including intermediate grades, for a total of nine grades as follows: 0 (absent), 0.5 (slight), I (mild), I.5 (between mild and moderate), 2 (moderate), 2.5 (between moderate and severe), 3 (severe), 3.5 (between severe and very severe), 4 (very severe). There were no significant differences between the two groups (Steel-Dwass test). a: control cream, b: test cream.

Abbreviation: $A D$, atopic dermatitis.

or above. However, both groups had a score of up to 2: moderate until week 4; this suggested that the remission period was being maintained. There were no significant differences in the results of the efficacy assessment.

During the study period, subjects recorded themselves daily for itching during the day and night. The results indicated the number of subjects who did not itch at all for a week (Table 1); in the control cream group, the number was almost the same from week 0 to week 4 . However, in the test cream group, the number of subjects who did not itch at all during the day and night at week 2-3 and week 3-4 were higher than those at week $0-1$.

\section{Changes of Skin Properties Value}

The skin hydration value and TEWL were measured to assess the improvement of skin properties. Each measurement was calculated and compared with the amount of change based on week 0 . The change in skin hydration was $148.1 \pm 21.3 \mu \mathrm{S}$ in

Table I The Number of Subjects Who Did Not Experience Itchiness Throughout the Study Period

\begin{tabular}{|l|l|l|l|l|l|}
\hline & & $\begin{array}{l}\text { Week } \\
\text { 0-I }\end{array}$ & $\begin{array}{l}\text { Week } \\
\text { I-2 }\end{array}$ & $\begin{array}{l}\text { Week } \\
\mathbf{2 - 3}\end{array}$ & $\begin{array}{l}\text { Week } \\
\mathbf{3 - 4}\end{array}$ \\
\hline $\begin{array}{l}\text { Control cream } \\
\text { Group }(\mathrm{n}=7)\end{array}$ & $\begin{array}{l}\text { Day } \\
\text { Night }\end{array}$ & $\begin{array}{l}3(43 \%) \\
3(43 \%)\end{array}$ & $\begin{array}{l}4(57 \%) \\
3(43 \%)\end{array}$ & $\begin{array}{l}3(43 \%) \\
4(57 \%)\end{array}$ & $\begin{array}{l}3(43 \%) \\
3(43 \%)\end{array}$ \\
\hline $\begin{array}{l}\text { Test cream } \\
\text { Group }(\mathrm{n}=10)\end{array}$ & Day & $4(40 \%)$ & $4(40 \%)$ & $7(70 \%)$ & $6(60 \%)$ \\
7 Night & $5(50 \%)$ & $6(60 \%)$ & $7(70 \%)$ & $7(70 \%)$ \\
\hline
\end{tabular}

Notes: The subjects filled in a journal regarding the presence or absence of itching during the day and night, every day during the study period. The number of subjects who did not experience itchiness in test cream group gradually increased during the study period. the test cream group at week 2 , and was significantly higher compared to the control cream group $(35.0 \pm 18.1 \mu \mathrm{S}$, mean \pm $\mathrm{SE}, \mathrm{p}<0.05)$ ) (Figure 6A). The change in TEWL was $-0.76 \pm$ $0.19 \mathrm{~g} / \mathrm{hm}^{2}$ in the test cream group at week 3 (Figure 6B). The change in TEWL was significantly lower in the test cream group than in the control cream group $\left(0.81 \pm 0.51 \mathrm{~g} / \mathrm{hm}^{2}, \mathrm{p}<\right.$ $0.05)$. The results indicated that the test cream has a more significant effect on the improvement of skin hydration and barrier function compared to the control cream.

\section{Safety Assessment}

No adverse events were caused by the test products. Furthermore, the test products were deemed safe based on the patient diary and the physician's findings.

\section{Discussion}

In the present study, both the test and control cream groups maintained the same level of remission during the study period (Figure 5). However, the number of subjects who did not experience itching for a week tended to increase in the test cream group (Table 1). Additionally, the skin hydration value was significantly higher in the test cream group. The TEWL value in the test cream group was significantly lower than in the control cream group (Figure 6). These results suggest that the test cream is highly effective at maintaining the remission period of $\mathrm{AD}$ and improving the skin barrier.

The heparinoid concentration reported to have a moisturizing effect was the same for both creams, but the improvement of skin function differed due to the lamellar structure of the test cream formulation. In the test cream, the dry coating film had a repeating structure of approximately $10 \mathrm{~nm}$, this was confirmed by small-and wide-angle X-ray scattering and TEM observation (Figures 2 and 3). It has been reported that the intercellular lipids in the human stratum corneum have a short periodic structure of $6 \mathrm{~nm}$ and long periodic structure of $13 \mathrm{~nm},{ }^{24}$ and display similarities with the structure of the dry coating formed by the heparinoid cream we developed.

The lamellar structure of the test cream may have contributed to the improvement of the skin barrier function and moisture content of the stratum corneum. Intercellular lipids in the stratum corneum play an important role in human skin. Intercellular lipids in the stratum corneum are released by the lamellar granules of epidermal cells to form a lamellar structure. ${ }^{25}$ In $\mathrm{AD}$, the size of the lamellar granules is small, and only a limited number are released to the stratum corneum. ${ }^{26}$ Furthermore, in $\mathrm{AD}$, there is not only a decrease in the amount of intercellular lipids but there is also a change 

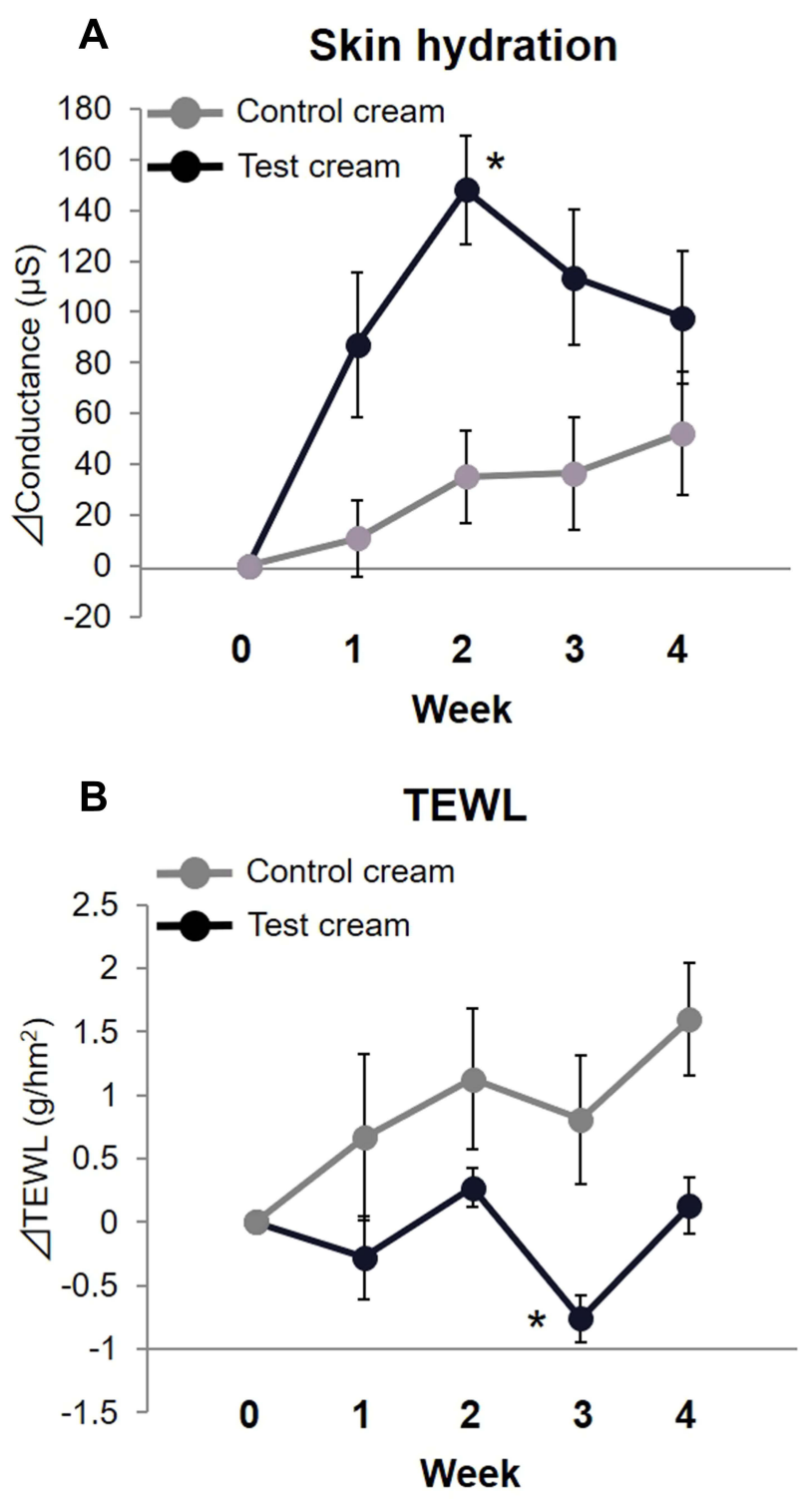

Figure 6 Changes in skin hydration and transepidermal water loss.

Notes: The changes of skin hydration value and transepidermal water loss from week 0 were shown. (A) The change in skin hydration value at week 2 in the test cream group was significantly higher compared to the control cream group (mean \pm $\mathrm{SE}, *_{\mathrm{p}}<0.05$, Steel-Dwass test). (B) The change of transepidermal water loss in the test cream group at week 3 was significantly lower compared to the control cream group (mean $\pm S E,{ }^{*} p<0.05$, Steel-Dwass test).

Abbreviations: TEWL, transepidermal water loss; SE, standard error

their composition, ${ }^{5}$ resulting in changes in the intercellular lipid structure of the stratum corneum of $\mathrm{AD} \cdot{ }^{27,28}$ In other words, a decrease in the amount of intercellular lipids and changes in their structure are major factors affecting the decrease in skin barrier function in $\mathrm{AD}$. In this study, TEWL levels were lower in the test cream group, although the value in the control cream group worsened over time (Figure 6). This may be because of the artificially constructed lamellar structures on the skin, which compensated for the fragile intercellular lipid structures associated with AD.
The moisture content of the stratum corneum is related to the amount of binding water incorporated into the lamellar structure, and is mainly composed of ceramides, which account for approximately $50 \%$ of the stratum corneum. The normal water content in the stratum corneum is $20-30 \%$, and the bound water stored in intercellular lipids is thought to play a vital role in moisture retention. ${ }^{29,30}$ The high level of water retention by a formulation which forms a lamellar structure containing pseudo-ceramide has been demonstrated in past reports. ${ }^{31}$ In this study, the high water retention observed in previous studies was maintained, and the moisture content was increased (Figure 6).

In our in vitro study, the test cream had a significantly lower level of V8 protease penetration compared to the control cream (Figure 4). This indicates that a formulation with a lamella structure may suppress the penetration of allergens. In $\mathrm{AD}$, itching is induced by the invasion of allergens, ${ }^{32}$ the ability of the test cream to inhibit allergens may have contributed to the decrease in the number of subjects who experienced itching (Table 1). In addition, the improvement in the moisturizing effect of the skin has been reported to reduce itchiness. Dry skin has nerve fibers related to itchiness, that are activated through physical or chemical stimulation, which extend into the epidermis. ${ }^{33}$ An increase in nerve fibers in the epidermis leaves a person vulnerable to external stimulation, causing itchiness. The application of a moisturizing agent has been shown to suppress epidermal nerve extension in a mouse dry skin model. ${ }^{34}$ Therefore, the test cream group not only directly suppressed the invasion of allergens but may also have reduced itching by improving the water content in the stratum corneum. In future, we should confirm whether the application of the heparinoid test cream helps reduce Th2 cytokines, such as IL-4, IL-13, IL-31, and TSLP etc., that are produced due to the invasion of allergens.

\section{Conclusion}

The cream with lamellar structures containing heparinoid and pseudo-ceramides maintained the remission period to the same extent as general heparinoid cream. The cream with the lamellar structure had a significant ability to suppress allergen penetration and improved the skin hydration and TEWL, which are important for skin barrier function during the remission period in $\mathrm{AD}$ patients. Thus, to treat $\mathrm{AD}$, which is referred to as a barrier disease, we believe that the heparinoid cream that forms a lamellar structure is 
suitable as a moisturizing agent, which is able to maintain the remission period and simultaneously improve skin health. The use of such a moisturizing agent during the remission period may help reduce the amount of steroidal drugs required. It may also be an effective moisturizer during the exacerbation period of AD.

\section{Abbreviations}

AD, atopic dermatitis; CBB, Coomassie brilliant blue; TEWL, transepidermal water loss; TEM, transmission electron microscopy; TSLP, thymic stromal lymphopoietin.

\section{Acknowledgments}

We would like to extend our gratitude to the staff at the Dermalabo Co., Ltd. for their considerable cooperation during the study.

\section{Disclosure}

The authors state no conflicts of interest in this work.

\section{References}

1. Katayama I, Aihara M, Ohya Y, et al. Japanese guidelines for atopic dermatitis 2017. Allergol Int. 2017;66(2):230-247. doi:10.1016/j. alit.2016.12.003

2. Takai T, Ikeda S. Barrier dysfunction caused by environmental proteases in the pathogenesis of allergic diseases. Allergol Int. 2011;60:25-35. doi:10.2332/allergolint.10-RAI-0273

3. Matsuki H, Kiyokane K, Matsuki T, et al. Recharacterization of the nonlesional dry skin in atopic dermatitis through disrupted barrier function. Exog Dermatol. 2004;3(6):282-292. doi:10.1159/000091909

4. Otsuka A, Nomura T, Rerknimitr P, et al. The interplay between genetic and environmental factors in the pathogenesis of atopic dermatitis. Immunoll Rev. 2017;278:246-262. doi:10.1111/imr.12545

5. Ishikawa J, Narita H, Kondo N, et al. Changes in the ceramide profile of atopic dermatitis patients. $J$ Invest Dermatol. 2010;130:2511-2514. doi:10.1038/jid.2010.161

6. Kobayashi T, Glatz M, Horiuchi K, et al. Dysbiosis and Staphylococcus aureus colonization drives inflammation in atopic dermatitis. Immunity. 2015;42:756-766. doi:10.1016/j.immuni.20 15.03.014

7. Soumelis V, Reche PA, Kanzler H, et al. Human epithelial cells trigger dendritic cell mediated allergic inflammation by producing TSLP. Nat Immunol. 2002;3:673-680. doi:10.1038/ni805

8. Mollannazar NK, Smith PK, Yosipovitch G. Mediators of chronic pruritus in atopic dermatitis: getting the itch out? Clin Rev Allergy Immunol. 2016;51:263-292. doi:10.1007/s12016-015-8488-5

9. Dillon SR, Sprecher C, Hammond A, et al. Interleukin 31, a cytokine produced by activated $\mathrm{T}$ cells, induces dermatitis in mice. Nat Immunol. 2004;5:752-760.

10. Wilson SR, The L, Batia LM, et al. The epithelial cell-derived atopic dermatitis cytokine TSLP activates neurons to produce itch. Cell. 2013;155:285-295. doi:10.1016/j.cell.2013.08.057

11. Nemoto O, Morikawa R. Topical maintenance therapy in atopic dermatitis of children: clinical usefulness of the heparinoid cream. Japan J Clin Dermatol. 2006;60:638-642.
12. Doi T, Ishii R, Hosokawa $\mathrm{S}$, Hirano $T$, Naruse T. Mechanisms of heparinoid-induced skin moisturizing effect: influence on bound water and lamellar structures in stratum corneum. Nishi Nihon Hifuka. 2007;69:44-50. doi:10.2336/nishinihonhifu.69.44

13. Matsuki H, Kiyokane K, Matsuki T, Sato S, Imokawa G. Reevaluation of the importance of barrier dysfunction in the nonlesional dry skin of atopic dermatitis patients through the use of two barrier creams. Exog Dermatol. 2004;3:293-302. doi:10.1159/000091910

14. Sajic D, Asiniwasis R, Skotnicki-Grant S. A look at epidermal barrier function in atopic dermatitis: physiologic lipid replacement and the role of ceramides. Skin Ther Lett. 2012;17:6-9.

15. Elias PM. Stratum corneum defensive functions: an integrated view. $J$ Invest Dermatol. 2005;125:183-200. doi:10.1111/j.0022202X.2005.23668.x

16. Leung DY, Jain N, Leo HL. New concepts in the pathogenesis of atopic dermatitis. Curr Opin Immunol. 2003;15:634-638. doi:10.1016/j.coi.2003.09.009

17. Jensen JM, Folster-Holst R, Baranowsky A, et al. Impaired sphingomyelinase activity and epidermal differentiation in atopic dermatitis. $J$ Invest Dermatol. 2004;122:1423-1431. doi:10.1111/j.0022202X.2004.22621.x

18. Toncic RJ, Jakasa I, Hadzavdic SL, et al. Altered levels of sphingosine, sphinganine and their ceramides in atopic dermatitis are related to skin barrier function, disease severity and local cytokine milieu. Int J Mol Sci. 2020;21:1958. doi:10.3390/ijms21061958

19. Macheleidt O, Kaiser H, Sandhoff K. Deficiency of epidermal protein-bound omega-hydroxyceramides in atopic dermatitis. $J \quad$ Invest Dermatol. 2002;119:166-173. doi:10.1046/j.15231747.2002.01833.x

20. Bouwstra JA, Ponec M. The skin barrier in healthy and diseased state. Biochim Biophys Acta. 2006;1758:2080-2095. doi:10.1016/j. bbamem.2006.06.021

21. Bouwstra JA, Gooris G, Ponec M. The lipid organization of the skin barrier: liquid and crystalline domains coexist in lamellar phases. J Biol Phys. 2002;28:211-223. doi:10.1023/A:1019983715589

22. Hatta I, Ohta N, Inoue K, Yagi N. Coexistence of two domains in intercellular lipid matrix of stratum corneum. Biochim Biophys Acta. 2006;1758:1830-1836. doi:10.1016/j.bbamem.2006.08.014

23. Seki H, Furue M, Furukawa F, et al. Guidelines for management of atopic dermatitis. J Dermatol. 2009;36:563-577. doi:10.1111/j.13468138.2009.00706.x

24. Hatta I. The state of the art and perspective of structural study on skin stratum corneum. Oleo science. 2012;12:25-32. doi:10.5650/ oleoscience. 12.25

25. Elias PM, Goerke J, Friend DS. Mammalian epidermal barrier layer lipids: composition and influence on structure. J Invest Dermatol. 1977;69:535-546. doi:10.1111/1523-1747.ep12687968

26. Fartasch M, Bassukas ID, Diepgen TL. Disturbed extruding mechanism of lamellar bodies in dry non-eczematous skin of atopics. Br J Dermatol. 1992;127:221-227. doi:10.1111/j.1365-2133.1992.tb00118.x

27. Janssens M, van Smeden J, Gooris GS, et al. Lamellar lipid organization and ceramide composition in the stratum corneum of patients with atopic eczema. J Invest Dermatol. 2011;131:2136-2138. doi:10.1038/ni1084

28. Janssens M, van Smeden J, Gooris GS, et al. Increase in short-chain ceramides correlates with an altered lipid organization and decreased barrier function in atopic eczema patients. $J$ Lipid Res. 2012;53:2755-2766. doi:10.1194/jlr.P030338

29. Moore DJ, Rawlings AV. The chemistry, function and (patho)physiology of stratum corneum barrier ceramides. Int J Cosmet Sci. 2017;39:366-372. doi:10.1111/ics.12399

30. Inoue $\mathrm{T}$, Tsujii $\mathrm{K}$, Okamoto $\mathrm{K}$, Toda $\mathrm{K}$. Differential scanning Calorimetric studies on the melting behavior of water in stratum corneum. J Invest Dermatol. 1986;86:689-693. doi:10.1111/15231747.ep12276282 
31. Orita M, Uchiyama M, Hanamoto $\mathrm{T}$, et al. Formation of pseudo-intercellular lipids membrane on the skin surface by the alpha-gel holding a large amount of water. J Soc Cosmet Chem Jpn. 2012;46:25-32. doi:10.5107/sccj.46.25

32. Mori T, Ishida K, Mukumoto S, et al. Comparison of skin barrier function and sensory nerve electric current perception threshold between IgE-high extrinsic and IgE-normal intrinsic types of atopic dermatitis. $\mathrm{Br} J$ Dermatol. 2010;162:83-90. doi:10.1111/j.13652133.2009.09440.x
33. Tominaga M, Takamori K. Itch and nerve fibers with special reference to atopic dermatitis: therapeutic implications. J Dermatol. 2014;41:205-212. doi:10.1111/1346-8138.12317

34. Kamo A, Tominaga M, Negi O, et al. Topical application of emollients prevents dry skin-inducible intraepidermal nerve growth in acetone-treated mice. J Dermatol Sci. 2011;62:64-71. doi:10.1016/j. jdermsci.2011.01.008

\section{Publish your work in this journal}

Clinical, Cosmetic and Investigational Dermatology is an international, peer-reviewed, open access, online journal that focuses on the latest clinical and experimental research in all aspects of skin disease and cosmetic interventions. This journal is indexed on CAS
The manuscript management system is completely online and includes a very quick and fair peer-review system, which is all easy to use. Visit http://www.dovepress.com/testimonials.php to read real quotes from published authors. 